\title{
Continuous Assessment of Cardiac Afterload Using Aortic Velocity - Pressure Loop Analysis in patients under general anesthesia: A Pilot Study
}

Le Gall A., Vallee F., Joachim J., Mateo J., Mebazaa A., Gayat E.

Hopital Lariboisière; Assistance publique - Hôpitaux de Paris, Dept of Anaesthesiology \& Intensive Care, Paris, France

\section{Introduction:}

Cardiac Afterload is determined by arterial resistance, arterial compliance and aortic waves reflection. We developed an integrative analysis of Aortic Velocity and Arterial Pressure (AP) signals, as Velocity/Pressure (Vel/Pre) Loops, that should allow a mini-invasive assessment of compliance and Aortic wave reflection. Our aim was to assess vasopressor-induced alterations in afterload during anesthesia.

\section{Materials and methods:}

20 patients were included. During anesthesia, cardiac output $(\mathrm{CO})$ and femoral AP were continuously recorded using CombiQ $^{\text {tm }}$ monitor (Deltex Medical, Chichester, UK). Both signals were plotted to display Vel/Pre Loop diagram. We derived 4 points: A (Diastolic Pressure), B (Pressure at maximal Velocity), C (Systolic Pressure), and D (Dicrotic Pressure). Two angles were described: $\beta$ and $\gamma$, between AC and $\mathrm{AB}$ lines, respectively, and horizontal axis. An arterial tonometer (Sphygmocor, Nellcor System@) was used to calculate Augmentation Index (AIx) and Total Arterial Compliance $($ Ctot $=$ Stroke Volume $/$ central Pulse Pressure) . When baseline Mean Arterial Pressure (MAP) decreased by 20\%, vasopressors were administered. Parameters were recorded before (T0) and at vasopressor's peak effect (T1).
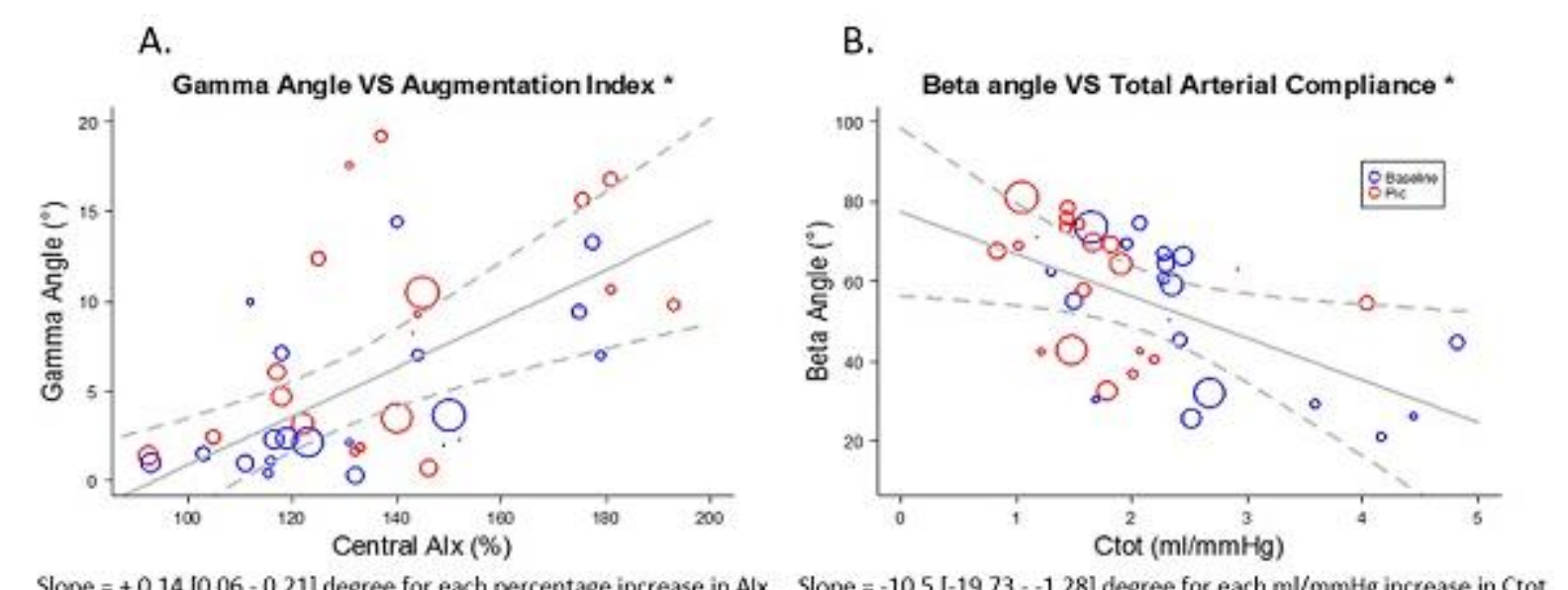

Figure 2. Meta regression for association between Cardiac afterload components and Vel/Pre Loop parameters. A. Association Between Gamma angle and Augmentation Index (Central Aix). B. Association between Beta Angle angle and Total Arterial
Compliance (Ctot). Samples were obtained both at baseline and at maximal effect of vasopressor agent used. Each 20 patient is represented by both blue and red circle. Radius of a circle represents the number of repeated measure for each patient. " $p<0.05$
A.

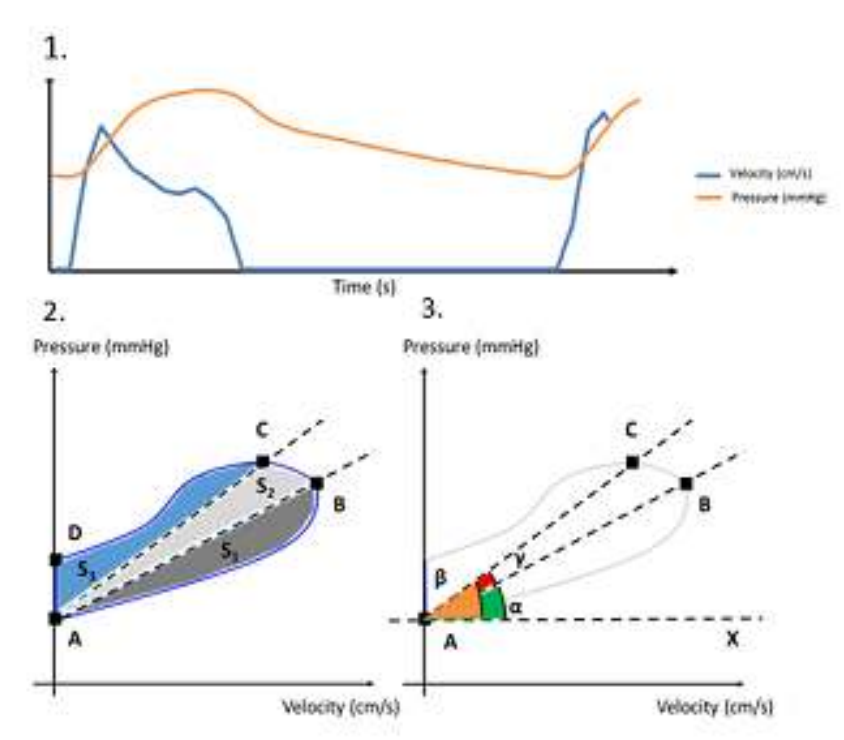

B.

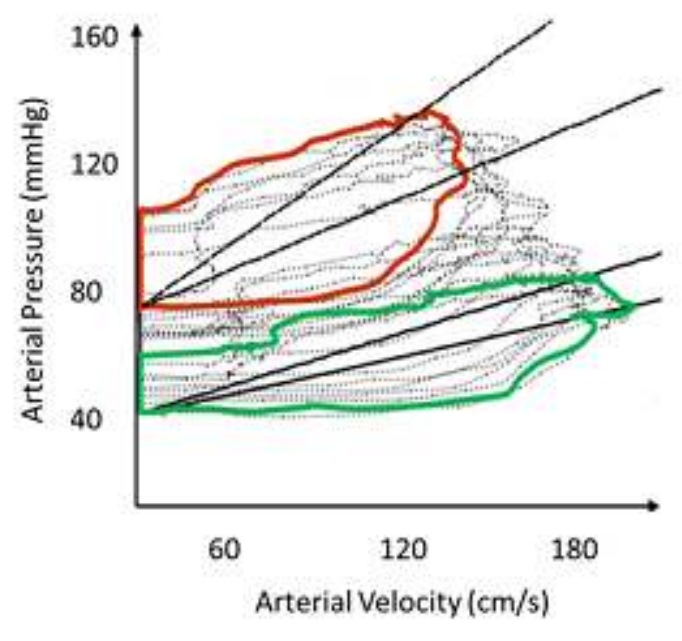

Figure 1. Examples of Vel/Pre Loops. A. Schematic representation of Vel/Pre Loop. 1. Time domain representation of Velocity and pressure signals. 2. Vel/Pre Loop displaying Surfaces (S1, S2, S3) delimited by A, B, C, and D points and the Vel/Pre Loop. 3. Vel Pre Loop diplaying Angles $(\alpha, \beta$ and v) delimited by $A B$ and $A C$ lines, and horizontal axis. B Example of a Vel/Pre Loop during vasopressor agent administration. Green Line: Baseline; Red Line : Maximal effect of the vasopressor.

\begin{abstract}
Results:
We studied 118 measurements. Between T0 and T1, increase in MAP (70 [66-73] vs 91 [84-92]mmHg; p< 0.001) was associated with decrease in $\mathrm{CO}(4.5[3.8-6.1]$ vs 3.8 [3.34.9]L/min; $\mathrm{p}<0.001)$, and Ctot $(2.3[1.9-2.6]$ vs 1.6 [1.4$1.9] \mathrm{ml} / \mathrm{mmHg} ; \mathrm{p}<0.001)$, as well as increase in AIx (127[116149] vs $138[124-153] \% ; \mathrm{p}=0.005), \beta\left(56[32-65]\right.$ vs $66[43-72]^{\circ}$; $\mathrm{p}<0.001)$ and $\gamma\left(2.3[1.4-7]\right.$ vs $\left.8.8[3-11.1]^{\circ} ; \mathrm{p}<0.001\right)$. An association was found between AIx and $\gamma$, and a negative association between Ctot and $\beta$.
\end{abstract}

\section{Conclusion:}

Vel/Pre Loop assessment could monitor cardiac afterload alterations in anesthetized patients, combining oesophageal Doppler and arterial pressure. 